\title{
On Fractional Derivatives and Primitives of Periodic Functions
}

\author{
I. Area, ${ }^{1}$ J. Losada, ${ }^{2}$ and J. J. Nieto ${ }^{2,3}$ \\ ${ }^{1}$ Departamento de Matemática Aplicada II, E.E. Telecomunicación, Universidade de Vigo, 36310 Vigo, Spain \\ ${ }^{2}$ Facultad de Matemáticas, Universidade de Santiago de Compostela, 15782 Santiago de Compostela, Spain \\ ${ }^{3}$ Faculty of Science, King Abdulaziz University, P.O. Box 80203, Jeddah 21589, Saudi Arabia
}

Correspondence should be addressed to J. J. Nieto; juanjose.nieto.roig@usc.es

Received 23 May 2014; Revised 8 July 2014; Accepted 8 July 2014; Published 14 August 2014

Academic Editor: Hari M. Srivastava

Copyright (c) 2014 I. Area et al. This is an open access article distributed under the Creative Commons Attribution License, which permits unrestricted use, distribution, and reproduction in any medium, provided the original work is properly cited.

We prove that the fractional derivative or the fractional primitive of a $T$-periodic function cannot be a $\widetilde{T}$-periodic function, for any period $\widetilde{T}$, with the exception of the zero function.

\section{Introduction}

Periodic functions $[1$, Ch. 3, pp. 58-92] play a central role in mathematics since the seminal works of Fourier $[2,3]$. Nowadays, periodic functions appear in applications ranging from electromagnetic radiation to blood flow and of course in control theory in linear time-varying systems driven by periodic input signals [4]. Linear time-varying systems driven by periodic input signals are ubiquitous in control systems, from natural sciences to engineering, economics, physics, and the life science $[4,5]$. Periodic functions also appear in automotive engine applications [6], optimal periodic scheduling of sensor networks $[7,8]$, or cyclic gene regulatory networks [9], to give some applications.

It is an obvious fact that the classical derivative, if it exists, of a periodic function is also a periodic function of the same period. Also the primitive of a periodic function may be periodic (e.g., $\cos t$ as primitive of $\sin t$ ).

The idea of integral or derivatives of noninteger order goes back to Riemann and Liouville $[3,10]$. Probably the first application of fractional calculus was made by Abel in the solution of the integral equation that arises in the formulation of the tautochrone problem [11]. Fractional calculus appears in many different contexts as speech signals, cardiac tissue electrode interface, theory of viscoelasticity, or fluid mechanics. The asymptotic stability of positive fractionalorder nonlinear systems has been proved in [12] by using the Lyapunov function. We do not intend to give a full list of applications but to show the wide range of them.
In this paper we prove that periodicity is not transferred by fractional integral or derivative, with the exception of the zero function. Although this property seems to be known $[10,13,14]$, in Section 3 we give a different proof by using the Laplace transform. Our approach relies on the classical concepts of fractional calculus and elementary analysis. Moreover, by using a similar argument as in [15], in Section 4 we prove that the fractional derivative or primitive of a $T$ periodic function cannot be $\widetilde{T}$-periodic for any period $\widetilde{T}$. A particular but nontrivial example is explicitly given. Finally, as a consequence we show in Section 5 that an autonomous fractional differential equation cannot have periodic solutions with the exception of constant functions.

\section{Preliminaries}

Let $T>0$. If $f: \mathbb{R} \rightarrow \mathbb{R}$ is $T$ periodic and $f \in \mathscr{C}^{1}(\mathbb{R})$, then the derivative $f^{\prime}$ is also $T$-periodic. However, the primitive of $f$

$$
F(t)=\int_{0}^{t} f(s) d s
$$

is not, in general, T-periodic. Just take $f(t)=1$ so that $F(t)=t$ is not $T^{\prime}$-periodic for any $T^{\prime}>0$. The necessary and sufficient condition for $F$ to be $T$-periodic is that

$$
\int_{0}^{T} f(s) d s=0 .
$$


The purpose of this note is to show that the fractional derivative or the fractional primitive of a $T$-periodic function cannot be $T$-periodic function with the exception, of course, of the zero function. We use the notation

$$
F=I^{1} f, \quad f^{\prime}=D^{1} f
$$

and note that

$$
D^{1}\left(I^{1} f\right)(t)=D^{1} F(t)=f(t)
$$

but

$$
I^{1}\left(D^{1} f\right)(t)=f(t)-f(0)
$$

and $I^{1}\left(D^{1} f\right)$ does not coincide with $f$ unless $f(0)=0$.

We recall some elements of fractional calculus. Let $\alpha \in$ $(0,1)$ and $f: \mathbb{R} \rightarrow \mathbb{R}$. We point out that $f$ is not necessarily continuous. The fractional integral of $f$ of order $\alpha$ is defined by [16]

$$
I^{\alpha} f(t)=\frac{1}{\Gamma(\alpha)} \int_{0}^{t}(t-s)^{\alpha-1} f(s) d s,
$$

provided the right-hand side is defined for a.e. $t \in \mathbb{R}$. If, for example, $f \in \mathscr{L}^{1}(\mathbb{R})$, then the fractional integral (6) is well defined and $I^{\alpha} f \in \mathscr{L}^{1}(0, T)$, for any $T>0$. Moreover, the fractional operator

$$
I^{\alpha}: \mathscr{L}^{1}(0, T) \longrightarrow \mathscr{L}^{1}(0, T)
$$

is linear and bounded.

The fractional Riemann-Liouville derivative of order $\alpha$ of $f$ is defined as $[16,17]$

$$
D^{\alpha} f(t)=D^{1} I^{1-\alpha} f(t)=\frac{1}{\Gamma(1-\alpha)} \frac{d}{d t} \int_{0}^{t}(t-s)^{-\alpha} f(s) d s .
$$

This is well defined if, for example, $f \in \mathscr{L}_{\text {loc }}^{1}(\mathbb{R})$.

There are many more fractional derivatives. We are not giving a complete list but recall the Caputo derivative $[16,17]$

$$
{ }^{c} D^{\alpha} f(t)=I^{1-\alpha} D^{1} f(t)=\frac{1}{\Gamma(1-\alpha)} \int_{0}^{t}(t-s)^{-\alpha} f^{\prime}(s) d s,
$$

which is well defined, for example, for absolutely continuous functions.

As in the integer case we have

$$
D^{\alpha}\left(I^{\alpha} f\right)(t)=f(t), \quad{ }^{c} D^{\alpha}\left(I^{\alpha} f\right)(t)=f(t)
$$

but $I^{\alpha}\left(D^{\alpha} f\right)$ or $I^{\alpha}\left({ }^{c} D^{\alpha} f\right)$ are not, in general, equal to $f$. Indeed

$$
I^{\alpha}\left({ }^{c} D^{\alpha} f\right)(t)=f(t)-f(0),
$$

and (see [17, (2.113), p. 71])

$$
I^{\alpha}\left(D^{\alpha} f\right)(t)=f(t)-\frac{D^{\alpha-1} f(0)}{\Gamma(\alpha)} t^{\alpha-1} .
$$

Also [16, (2.4.4), p. 91]

$$
{ }^{c} D^{\alpha} f(t)=D^{\alpha}(f(t)-f(0)) .
$$

\section{The Fractional Derivative or Primitive of a $T$-Periodic Function Cannot Be T-Periodic}

We prove the following result in Section 3.1 below.

Theorem 1. Let $f: \mathbb{R} \rightarrow \mathbb{R}$ be a nonzero $T$-periodic function with $f \in \mathscr{L}_{\text {loc }}^{1}(\mathbb{R})$. Then $I^{\alpha} f$ cannot be T-periodic for any $\alpha \in$ $(0,1)$.

Corollary 2. Let $f: \mathbb{R} \rightarrow \mathbb{R}$ be a nonzero $T$-periodic function such that $f \in \mathscr{L}_{\text {loc }}^{1}(\mathbb{R})$. Then the Caputo derivative ${ }^{c} D^{\alpha} f$ cannot be $T$-periodic for any $\alpha \in(0,1)$. The same result holds for the fractional derivative $D^{\alpha} f$.

Proof. Suppose that ${ }^{c} D^{\alpha} f$ is $T$-periodic. Then by Theorem 1 , $I^{\alpha}\left({ }^{c} D^{\alpha} f\right)$ cannot be $T$-periodic. However,

$$
I^{\alpha}\left({ }^{c} D^{\alpha} f\right)(t)=f(t)-f(0)
$$

is $T$-periodic. In relation to the fractional Riemann-Liouville derivative, suppose that $D^{\alpha} f$ is $T$-periodic and consider the function $\widehat{f}=f-f(0)$ which is also $T$-periodic. Then

$$
{ }^{c} D^{\alpha} \widehat{f}=D^{\alpha} \widehat{f}
$$

cannot be $T$-periodic.

3.1. Proof of Theorem 1. Let $\alpha \in(0,1)$ and $T>0$. By reduction to the absurd, in this section we suppose that $I^{\alpha} f$ is $T$ periodic. Then

$$
I^{\alpha} f(0)=0=I^{\alpha} f(T)
$$

that is,

$$
\int_{0}^{T}(T-s)^{\alpha-1} f(s) d s=0
$$

Lemma 3. Assume $f \in \mathscr{L}_{\text {loc }}^{1}(\mathbb{R})$ is T-periodic. If $I^{\alpha} f$ is also T-periodic, then

$$
\int_{0}^{T}(n T-s)^{\alpha-1} f(s) d s=0, \quad(n \in \mathbb{N}:=\{1,2,3, \ldots\}) .
$$


Proof. For $n=1$ the latter equality reduces to (17). For $n=2$,

$$
\begin{aligned}
0= & I^{\alpha} f(2 T) \\
= & \frac{1}{\Gamma(\alpha)} \int_{0}^{2 T}(2 T-s)^{\alpha-1} f(s) d s \\
= & \frac{1}{\Gamma(\alpha)} \int_{0}^{T}(2 T-s)^{\alpha-1} f(s) d s \\
& +\frac{1}{\Gamma(\alpha)} \int_{T}^{2 T}(2 T-s)^{\alpha-1} f(s) d s \\
= & \frac{1}{\Gamma(\alpha)} \int_{0}^{T}(2 T-s)^{\alpha-1} f(s) d s \\
& +\frac{1}{\Gamma(\alpha)} \int_{0}^{T}(T-r)^{\alpha-1} f(r+T) d r \\
= & \frac{1}{\Gamma(\alpha)} \int_{0}^{T}(2 T-s)^{\alpha-1} f(s) d s \\
& +\frac{1}{\Gamma(\alpha)} \int_{0}^{T}(T-r)^{\alpha-1} f(r) d r \\
= & \frac{1}{\Gamma(\alpha)} \int_{0}^{T}(2 T-s)^{\alpha-1} f(s) d s .
\end{aligned}
$$

The proof follows by induction on $n$. Assume that (18) is valid for some $n \in \mathbb{N}$. Then

$$
\begin{aligned}
& \int_{0}^{(n+1) T}((n+1) T-s)^{\alpha-1} f(s) d s \\
& \quad=\sum_{j=0}^{n} \int_{j T}^{(j+1) T}((n+1) T-s)^{\alpha-1} f(s) d s,
\end{aligned}
$$

and, by periodicity,

$$
\int_{0}^{(n+1) T}((n+1) T-s)^{\alpha-1} f(s) d s=I^{\alpha} f((n+1) T)=0 .
$$

Moreover, for $j=1,2, \ldots, n$,

$$
\begin{aligned}
\sum_{j=1}^{n} & \int_{j T}^{(j+1) T}((n+1) T-s)^{\alpha-1} f(s) d s \\
& =\sum_{j=1}^{n} \int_{0}^{T}((n+1-j) T-r)^{\alpha-1} f(r) d r=0
\end{aligned}
$$

by hypothesis of induction since $1 \leq n+1-j \leq n$. Hence,

$$
\begin{aligned}
0 & =\sum_{j=0}^{n} \int_{j T}^{(j+1) T}((n+1) T-s)^{\alpha-1} f(s) d s \\
& =\int_{0}^{T}((n+1) T-s)^{\alpha-1} f(s) d s .
\end{aligned}
$$

Lemma 4. Under the hypothesis of Lemma 3,

$$
\int_{0}^{T} f(s) d s=0
$$

Proof. Let $f^{+}$and $f^{-}$be the positive and negative parts of $f$,

$$
\begin{gathered}
f^{+}(x)=\max (f(x), 0), \\
f^{-}(x)=-\min (f(x), 0), \\
f=f^{+}-f^{-} .
\end{gathered}
$$

Equation (18) implies that

$$
\int_{0}^{T}(n T-s)^{\alpha-1} f^{+}(s) d s=\int_{0}^{T}(n T-s)^{\alpha-1} f^{-}(s) d s .
$$

If $\int_{0}^{T} f^{+}(s) d s=0$ or $\int_{0}^{T} f^{-}(s) d s=0$, then from (18) we get $f=0$. We consider the case

$$
\int_{0}^{T} f^{+}(s) d s>\int_{0}^{T} f^{-}(s) d s>0 .
$$

For $n$ large

$$
\left(\frac{n T}{(n-1) T}\right)^{\alpha-1}>\frac{\int_{0}^{T} f^{-}(s) d s}{\int_{0}^{T} f^{+}(s) d s}
$$

or equivalently

$$
(n T)^{\alpha-1} \int_{0}^{T} f^{+}(s) d s>((n-1) T)^{\alpha-1} \int_{0}^{T} f^{-}(s) d s .
$$

Hence,

$$
\begin{aligned}
0 & =\int_{0}^{T}(n T-s)^{\alpha-1} f(s) d s \\
& \geq(n T)^{\alpha-1} \int_{0}^{T} f^{+}(s) d s-((n-1) T)^{\alpha-1} \int_{0}^{T} f^{-}(s) d s>0,
\end{aligned}
$$

which is a contradiction.

The case

$$
\int_{0}^{T} f^{-}(s) d s>\int_{0}^{T} f^{+}(s) d s>0
$$

is analogous.

Therefore,

$$
\begin{gathered}
\int_{0}^{T} f^{-}(s) d s=\int_{0}^{T} f^{+}(s) d s>0 \\
\int_{0}^{T} f(s) d s=0 .
\end{gathered}
$$


Lemma 5. Under the hypothesis of Lemma 3,

$$
\int_{0}^{T}(T+\delta-s)^{\alpha-1} f(s) d s=0, \quad \forall \delta \in[0, T]
$$

Proof. If $\delta=0$ and $\delta=T$, the equation reduces to (17) and (18), respectively. Let $0<\delta<T$.

$$
\begin{aligned}
I^{\alpha} f(T+\delta)= & \frac{1}{\Gamma(\alpha)} \int_{0}^{T+\delta}(T+\delta-s)^{\alpha-1} f(s) d s \\
= & \frac{1}{\Gamma(\alpha)} \int_{0}^{T}(T+\delta-s)^{\alpha-1} f(s) d s \\
& +\frac{1}{\Gamma(\alpha)} \int_{T}^{T+\delta}(T+\delta-s)^{\alpha-1} f(s) d s \\
= & \frac{1}{\Gamma(\alpha)} \int_{0}^{T}(T+\delta-s)^{\alpha-1} f(s) d s \\
& +\frac{1}{\Gamma(\alpha)} \int_{0}^{\delta}(\delta-r)^{\alpha-1} f(r+T) d r \\
= & \frac{1}{\Gamma(\alpha)} \int_{0}^{T}(T+\delta-s)^{\alpha-1} f(s) d s+I^{\alpha} f(\delta)
\end{aligned}
$$

By using the periodicity of $I^{\alpha} f$ we get (33).

Lemma 6. Under the hypothesis of Lemma 3,

$$
\int_{0}^{T}(T+t-s)^{\alpha-1} f(s) d s=0, \quad \forall t \in \mathbb{R} .
$$

Proof. For $t \in[0, T]$ or $t=n T, n=1,2, \ldots$, relation (35) is true. Let $t=n T+\delta$, so that $T+t=(n+1) T+\delta$. Then

$$
\begin{aligned}
I^{\alpha} f(\delta) & =I^{\alpha} f(T+t) \\
& =\frac{1}{\Gamma(\alpha)} \int_{0}^{(n+1) T+\delta}((n+1) T+\delta-s)^{\alpha-1} f(s) d s .
\end{aligned}
$$

Now, using the additive property of the integral, we have

$$
\begin{aligned}
& \frac{1}{\Gamma(\alpha)} \int_{0}^{(n+1) T+\delta}((n+1) T+\delta-s)^{\alpha-1} f(s) d s \\
& =\frac{1}{\Gamma(\alpha)} \sum_{j=0}^{n} \int_{j T}^{(j+1) T}((n+1) T+\delta-s)^{\alpha-1} f(s) d s \\
& \quad+\frac{1}{\Gamma(\alpha)} \int_{(n+1) T}^{(n+1) T+\delta}((n+1) T+\delta-s)^{\alpha-1} f(s) d s
\end{aligned}
$$

Let us compute separately the integrals in the right-hand side. In all the integrals depending on $j$, we use the (linear) change of variable $r=s-j T$ and rename $t^{\prime}=(n-j) T+\delta$ to obtain

$$
\begin{aligned}
& \int_{j T}^{(j+1) T}(n T+T+\delta-s)^{\alpha-1} f(s) d s \\
& \quad=\int_{0}^{T}(T+(n-j) T+\delta-r)^{\alpha-1} f(r+j T) d r \\
& \quad=\int_{0}^{T}\left(T+t^{\prime}-s\right)^{\alpha-1} f(s) d s .
\end{aligned}
$$

For the last integral we use the (linear) change of variable $r=$ $s-(n+1) T$ to get

$$
\begin{aligned}
& \int_{(n+1) T}^{(n+1) T+\delta}((n+1) T+\delta-s)^{\alpha-1} f(s) d s \\
& \quad=\int_{0}^{\delta}(\delta-r)^{\alpha-1} f(r+(n+1) T) d r=I^{\alpha} f(\delta) .
\end{aligned}
$$

By induction on $n$, as in Lemma 3, the proof follows.

Lemma 7. Let $f$ be a continuous and T-periodic function, $T>$ 0 . Let $0<\alpha<1$ be fixed. Assuming that

$$
\begin{aligned}
\int_{0}^{T}(T-s+t)^{\alpha-1} f(s) & =0, \quad \forall t \in \mathbb{R}, \\
\int_{0}^{T} f(s) d s & =0,
\end{aligned}
$$

then $f \equiv 0$.

Proof. Since $\int_{0}^{T} f(s) d s=0$ then $0=\int_{0}^{T} f(s) d s=\int_{0}^{T}\left(f^{+}(s)-\right.$ $\left.f^{-}(s)\right) d s$ and therefore we can define $c=\int_{0}^{T} f^{+}(s) d s=$ $\int_{0}^{T} f^{-s}(s) d s>0$. If $c=0$ then $f=0$.

Let us define

$$
\phi(t)=\int_{0}^{T}(T-s+t)^{\alpha-1} f(s) d s .
$$

From the hypothesis, we have that $\phi(t)=0$ at any $t \in \mathbb{R}$. Therefore, its integral is also zero. Let us integrate with respect to $t$ from $a$ to $b$ for $0 \leq a \leq b \leq T$

$$
\begin{aligned}
0 & =\int_{a}^{b} \phi(t) d t=\int_{a}^{b}\left(\int_{0}^{T}(T-s+t)^{\alpha-1} f(s) d s\right) d t \\
& =\int_{0}^{T}\left(\int_{a}^{b}(T-s+t)^{\alpha-1} d t\right) f(s) d s \\
& =\int_{0}^{T}\left(\frac{(b-s+T)^{\alpha}-(a-s+T)^{\alpha}}{\alpha}\right) f(s) d s,
\end{aligned}
$$

where we have assumed $0 \leq a<b, s<T$. Thus,

$$
\int_{0}^{T}\left[(b-s+T)^{\alpha}-(a-s+T)^{\alpha}\right] f(s) d s=0
$$


which implies that

$$
\psi(t)=\int_{0}^{T}(T-s+t)^{\alpha} f(s) d s
$$

is a constant function.

Moreover, since

$$
\begin{aligned}
t^{\alpha} c-(T+t)^{\alpha} c & \leq \int_{0}^{T}(T-s+t)^{\alpha} f(s) d s \\
& \leq(T+t)^{\alpha} c-t^{\alpha} c,
\end{aligned}
$$

where

$$
c=\int_{0}^{T} f^{+}(s) d s=\int_{0}^{T} f^{-}(s) d s
$$

in view of (24) and

$$
\lim _{t \rightarrow+\infty}\left((T+t)^{\alpha}-t^{\alpha}\right)=0
$$

we have that

$$
\int_{0}^{T}(T-s+t)^{\alpha} f(s) d s=0, \quad \forall t \in \mathbb{R} .
$$

Let

$$
\tilde{f}=f \cdot \chi_{[0, T]}, \quad \tilde{f}(t)= \begin{cases}f(t), & t \in[0, T] \\ 0, & t>T .\end{cases}
$$

If we define

$$
\varphi(t)=(T+t)^{\alpha}
$$

then the convolution of $\varphi$ and $\widetilde{f}$ is given by

$$
\begin{aligned}
(\varphi * \tilde{f}) & =\int_{0}^{+\infty} \varphi(t-s) \tilde{f}(s) d s \\
& =\int_{0}^{T}(T+t-s)^{\alpha} f(s) d s=0 .
\end{aligned}
$$

Therefore, if we apply the Laplace transform [18, Chapter 17] to the above equality it yields

$$
\mathscr{L}[\varphi * \tilde{f}]=\mathscr{L}[\varphi] \mathscr{L}[\tilde{f}]=\mathscr{L}[0]=0 .
$$

Since

$$
\mathscr{L}[\varphi]=s^{-\alpha-1} e^{s T} \Gamma(\alpha+1, s T),
$$

where $\Gamma(a, z)$ denotes the incomplete gamma function $[19$, Section 6.5], then $\mathscr{L}[\varphi] \neq 0$ which implies that $\mathscr{L}[\tilde{f}]=0$ and therefore $\widetilde{f}=0$, that is, $f=0$, on $[0, T]$.

\section{The Fractional Derivative or Primitive of a $T$-Periodic Function Cannot Be $\widetilde{T}$-Periodic for any Period $\widetilde{T}$}

Let $f$ be a $T$-periodic function and consider $u$ such that

$$
{ }^{c} D^{\alpha} u=f(t), \quad 0<\alpha<1 .
$$

Then

$$
u(t)=u(0)+I^{\alpha} f(t)
$$

and therefore

$$
\mathscr{L}[u(t)]=\mathscr{L} u_{0}+\mathscr{L}\left[I^{\alpha} f(t)\right]
$$

Let us assume that $u$ is a $\widetilde{T}$-periodic function. Then by using some basic properties of the Laplace transform it yields

$$
\frac{\int_{0}^{\widetilde{T}} u(t) \exp (-\lambda t) d t}{1-\exp (-\lambda \widetilde{T})}=\frac{u_{0}}{\lambda}+\frac{1}{\lambda^{\alpha}} \frac{\int_{0}^{T} f(t) \exp (-\lambda t) d t}{1-\exp (-\lambda T)}
$$

Therefore,

$$
\begin{aligned}
\lambda(1-\exp (-\lambda T)) \int_{0}^{\widetilde{T}} u(t) \exp (-\lambda t) d t \\
=u_{0}(1-\exp (-\lambda T))(1-\exp (-\lambda \widetilde{T})) \\
\quad+\lambda^{1-\alpha}(1-\exp (-\lambda \widetilde{T})) \int_{0}^{T} f(t) \exp (-\lambda t) d t .
\end{aligned}
$$

Let us consider $v=u-u_{0}$ so that $v$ is also $\widetilde{T}$-periodic and $v(0)=0$. The above equality becomes

$$
\begin{aligned}
& \lambda(1-\exp (-\lambda T)) \int_{0}^{\widetilde{T}} v(t) \exp (-\lambda t) d t \\
& \quad=\lambda^{1-\alpha}(1-\exp (-\lambda \widetilde{T})) \int_{0}^{T} f(t) \exp (-\lambda t) d t
\end{aligned}
$$

or equivalently

$$
\begin{aligned}
& \lambda^{\alpha} \frac{(1-\exp (-\lambda T))}{(1-\exp (-\lambda \widetilde{T}))} \int_{0}^{\widetilde{T}} v(t) \exp (-\lambda t) d t \\
& \quad=\int_{0}^{T} f(t) \exp (-\lambda t) d t
\end{aligned}
$$

Thus,

$$
\begin{gathered}
\frac{(1-\exp (-\lambda T))}{(1-\exp (-\lambda \widetilde{T}))} \sum_{i=0}^{\infty}(-1)^{i} \frac{\lambda^{\alpha+i}}{i !} \int_{0}^{\widetilde{T}} v(t) t^{i} d t \\
=\sum_{i=0}^{\infty}(-1)^{i} \frac{\lambda^{i}}{i !} \int_{0}^{T} f(t) t^{i} d t .
\end{gathered}
$$


Since

$$
\lim _{\lambda \rightarrow 0^{+}} \frac{(1-\exp (-\lambda T))}{(1-\exp (-\lambda \widetilde{T}))}=\frac{T}{\widetilde{T}}, \quad \lim _{\lambda \rightarrow 0^{+}} \lambda^{\alpha+i}=0,
$$

by using $0<\alpha<1$ and $i \geq 0$, the limit as $\lambda \rightarrow 0^{+}$of the left-hand side is zero, which implies

$$
\int_{0}^{T} f(t) d t=0
$$

Then

$$
\begin{aligned}
& \frac{(1-\exp (-\lambda T))}{(1-\exp (-\lambda \widetilde{T}))} \sum_{i=0}^{\infty}(-1)^{i} \frac{\lambda^{i}}{i !} \int_{0}^{\widetilde{T}} v(t) t^{i} d t \\
& =\lambda^{-\alpha} \sum_{i=1}^{\infty}(-1)^{i} \frac{\lambda^{i}}{i !} \int_{0}^{T} f(t) t^{i} d t \\
& =\lambda^{1-\alpha} \sum_{i=0}^{\infty}(-1)^{i+1} \frac{\lambda^{i}}{(i+1) !} \int_{0}^{T} f(t) t^{i+1} d t .
\end{aligned}
$$

If we consider $\lambda \rightarrow 0^{+}$in the latter expression we get

$$
\frac{T}{\widetilde{T}} \int_{0}^{\widetilde{T}} v(t) d t=0
$$

and therefore

$$
\int_{0}^{\widetilde{T}} v(t) d t=0
$$

By induction, we obtain that

$$
\int_{0}^{T} f(t) t^{i} d t=0, \quad \int_{0}^{\widetilde{T}} v(t) t^{i} d t=0, \quad i=0,1,2, \ldots
$$

Therefore, $f=u=0$ and there are no nonzero $\widetilde{T}$-periodic $L^{\infty}$-solutions of the problem.

Example 8. Let $f(t)=\sin (t)$ and $0<\alpha<1$. The Caputofractional derivative of $f(t)$ is given by

$$
{ }^{c} D^{\alpha} f(t)=\frac{t^{1-\alpha}}{\Gamma(2-\alpha)}{ }_{1} F_{2}\left(1 ; \frac{3-\alpha}{2}, 1-\frac{\alpha}{2} ;-\frac{t^{2}}{4}\right),
$$

where the hypergeometric series ${ }_{1} F_{2}(a ; b, c ; d)$ is defined as $([20,21]$, Chapter 15)

$$
{ }_{1} F_{2}(a ; b, c ; d)=\sum_{j=0}^{\infty} \frac{(a)_{j}}{j !(b)_{j}(c)_{j}} d^{j}
$$

and the Pochhammer symbol $(A)_{j}=A(A+1) \cdots(A+j-1)$, with $(A)_{0}=1$.
Since

$$
\begin{aligned}
& \frac{{ }^{c} D^{\alpha} f(\pi)}{{ }^{c} D^{\alpha} f(\pi+\widetilde{T})} \\
& =\pi^{1-\alpha}(\widetilde{T}+\pi)^{\alpha-1}{ }_{1} F_{2}\left(1 ; 1-\frac{\alpha}{2}, \frac{3}{2}-\frac{\alpha}{2} ;-\frac{\pi^{2}}{4}\right) \\
& \quad \times\left({ }_{1} F_{2}\left(1 ; 1-\frac{\alpha}{2}, \frac{3}{2}-\frac{\alpha}{2} ;-\frac{1}{4}(\widetilde{T}+\pi)^{2}\right)\right)^{-1}, \\
& \frac{{ }^{c} D^{\alpha} f(\pi / 2)}{{ }^{c} D^{\alpha} f(\pi / 2+\widetilde{T})} \\
& =\left(\frac{2 T}{\pi}+1\right){ }_{1}^{\alpha-1} F_{2}\left(1 ; 1-\frac{\alpha}{2}, \frac{3}{2}-\frac{\alpha}{2} ;-\frac{\pi^{2}}{16}\right) \\
& \quad \times\left({ }_{1} F_{2}\left(1 ; 1-\frac{\alpha}{2}, \frac{3}{2}-\frac{\alpha}{2} ;-\frac{1}{16}(2 T+\pi)^{2}\right)\right)^{-1},
\end{aligned}
$$

we have that ${ }^{c} D^{\alpha} f(t)$ is not a $\widetilde{T}$-periodic function for any positive $\widetilde{T}$ and $\alpha \in(0,1)$. Plotting both functions $\sin (t)$ and ${ }^{c} D^{\alpha} \sin (t)$, this last function seems to be periodic but it is not according to our results. Notice that Kaslik and Sivasundaram [10] gave the following alternate representation:

$$
{ }^{c} D^{\alpha} \sin (t)=\frac{1}{2} t^{1-\alpha}\left[E_{1,2-\alpha}(i t)+E_{1,2-\alpha}(-i t)\right],
$$

in terms of the two-parameter Mittag-Leffler function ([20, 21], Chapter 10)

$$
E_{\alpha, \beta}(z)=\sum_{k=0}^{\infty} \frac{z^{k}}{\Gamma(\alpha k+\beta)}
$$

\section{Periodic Solutions of Fractional Differential Equations}

In this section we show how Theorem 1 can be used to give a nonexistence result of periodic solutions for fractional differential equations.

Consider the first order ordinary differential equation

$$
D^{1} u(t)=\varphi(u(t)), \quad t \in \mathbb{R},
$$

where $\varphi: \mathbb{R} \rightarrow \mathbb{R}$ is continuous. An important question is the existence of periodic solutions [22-24].

If $u: \mathbb{R} \rightarrow \mathbb{R}$ is a $T$-periodic solution of (73) then obviously

$$
u(0)=u(T) .
$$

One can find $T$-periodic solutions of (73) by solving the equation only on the interval $[0, T]$ and then checking the values $u(0)$ and $u(T)$. If (74) holds, then extending by $T$ periodicity the function $u(t), t \in[0, T]$, to $\mathbb{R}$ we have a $T$ periodic solution of (73).

However, this is not possible for a fractional differential equation. Consider, for $\alpha \in(0,1)$, the equation

$$
{ }^{c} D^{\alpha} u(t)=\varphi(u(t)), \quad t \in \mathbb{R} .
$$


If $u$ is a solution of (75), let $f(t)=\varphi(u(t))$. Then

$$
u(t)=u(0)+I^{\alpha} f(t) .
$$

In the case that $u$ is a $T$-periodic solution of (75) we have that $f$ is also $T$-periodic. According to Theorem $1, I^{\alpha} f$ cannot be $T$-periodic unless it is the zero function and we have the following relevant result.

Theorem 9. The fractional equation (75) cannot have periodic solutions with the exception of constant functions $u(t)=u_{0}$, $t \in \mathbb{R}$, with $\varphi\left(u_{0}\right)=0$.

Remark 10. It is possible to consider the periodic boundary value problem

$$
\begin{gathered}
{ }^{c} D^{\alpha} u(t)=\varphi(u(t)), \quad t \in[0, T], \\
u(0)=u(T),
\end{gathered}
$$

as in, for example, [25], but one cannot extend the solution of that periodic boundary value problem on $[0, T]$ to a $T$ periodic solution on $\mathbb{R}$ (unless $u$ is a constant function, as indicated in Theorem 9).

Remark 11. The same applies to the Riemann-Liouville fractional differential equation

$$
D^{\alpha} u(t)=\varphi(u(t)), \quad t \in \mathbb{R}
$$

taking into account that

$$
\lim _{t \rightarrow 0^{+}} t^{1-\alpha} u(t)=\frac{D^{\alpha-1} u(0)}{\Gamma(\alpha)} .
$$

Example 12. Considering the fractional equation

$$
{ }^{c} D^{\alpha} u(t)=\psi(t, u(t)), \quad t \in \mathbb{R},
$$

with $\psi: \mathbb{R}^{2} \rightarrow \mathbb{R}$ defined by

$$
\begin{aligned}
& \psi(t, u) \\
& =u+\frac{t^{1-\alpha}}{\Gamma(2-\alpha)}{ }_{1} F_{2}\left(1 ; \frac{3-\alpha}{2}, 1-\frac{\alpha}{2} ;-\frac{t^{2}}{4}\right)-\sin (t),
\end{aligned}
$$

we have that $u(t)=\sin (t)$ is a $2 \pi$-periodic solution of $(80)$. This shows that the result of Theorem 9 is not valid for a nonautonomous fractional differential equation as (80).

\section{Conclusion}

By using the classical concepts of fractional calculus and elementary analysis, we have proved that periodicity is not transferred by fractional integral or derivative, with the exception of the zero function. We have also proved that the fractional derivative or primitive of a $T$-periodic function cannot be $\widetilde{T}$-periodic for any period $\widetilde{T}$. As a consequence we have showed that an autonomous fractional differential equation cannot have periodic solutions with the exception of constant functions.

\section{Conflict of Interests}

The authors declare that there is no conflict of interests regarding the publication of this paper.

\section{Acknowledgments}

The referees and editor deserve special thanks for careful reading and many useful comments and suggestions which have improved the paper. The work of I. Area has been partially supported by the Ministerio de Economía y Competitividad of Spain under Grant MTM2012-38794-C02-01, cofinanced by the European Community fund FEDER. J. J. Nieto also acknowledges partial financial support by the Ministerio de Economía y Competitividad of Spain under Grant MTM2010-15314, cofinanced by the European Community fund FEDER.

\section{References}

[1] K. Knopp, Theory of Functions Parts I and II, Two Volumes Bound as One, Part II, Dover Publications, 1996.

[2] W. E. Boyce and R. C. DiPrima, Elementary Differential Equations and Boundary Value Problems, John Wiley \& Sons, New York, NY. USA, 1965.

[3] J. Fourier, The Analytical Theory of Heat, Dover, New York, NY. USA, 1955.

[4] S. Bittanti and P. Colaneri, Periodic Systems. Filtering and Control, Springer, London, UK, 2009.

[5] J. Chauvin and N. Petit, "Periodic inputs reconstruction of partially measured linear periodic systems," Automatica, vol. 48, no. 7, pp. 1467-1472, 2012.

[6] J. Chauvin, G. Corde, N. Petit, and p. Rouchon, "Periodic input estimation for linear periodic systems: automotive engine applications," Automatica, vol. 43, no. 6, pp. 971-980, 2007.

[7] D. Shi and T. Chen, "Approximate optimal periodic scheduling of multiple sensors with constraints," Automatica, vol. 49, no. 4, pp. 993-1000, 2013.

[8] D. Shi and T. Chen, "Optimal periodic scheduling of sensor networks: a branch and bound approach," Systems \& Control Letters, vol. 62, no. 9, pp. 732-738, 2013.

[9] Y. Hori, T.-H. Kim, and S. Hara, "Existence criteria of periodic oscillations in cyclic gene regulatory networks," Automatica, vol. 47, no. 6, pp. 1203-1209, 2011.

[10] E. Kaslik and S. Sivasundaram, "Non-existence of periodic solutions in fractional-order dynamical systems and a remarkable difference between integer and fractional-order derivatives of periodic functions," Nonlinear Analysis: Real World Applications, vol. 13, no. 3, pp. 1489-1497, 2012.

[11] J. L. Lagrange, "Sue les courbes tautochrones," in Section deuxième: Mémoires extraits des recueils de l'Academie royale des sciences et Belles-Lettres de Berlin, vol. 2, pp. 317-332, GauthierVillars, Paris, France, 1868, Mém. de l'Acad. Roy. des Sci. et Belles-Lettres de Berlin 21, 1765.

[12] J. Shen and J. Lam, "Non-existence of finite-time stable equilibria in fractional-order nonlinear systems," Automatica, vol. 50, no. 2, pp. 547-551, 2014.

[13] M. S. Tavazoei, "A note on fractional-order derivatives of periodic functions," Automatica, vol. 46, no. 5, pp. 945-948, 2010. 
[14] M. S. Tavazoei and M. Haeri, "A proof for non existence of periodic solutions in time invariant fractional order systems," Automatica, vol. 45, no. 8, pp. 1886-1890, 2009.

[15] J. Wang, M. Fečkan, and Y. Zhou, "Nonexistence of periodic solutions and asymptotically periodic solutions for fractional differential equations," Communications in Nonlinear Science and Numerical Simulation, vol. 18, no. 2, pp. 246-256, 2013.

[16] A. A. Kilbas, H. M. Srivastava, and J. J. Trujillo, Theory and Applications of Fractional Differential Equations, vol. 204 of North-Holland Mathematics Studies, Elsevier Science, Amsterdam, The Netherlands, 2006.

[17] I. Podlubny, Fractional Differential Equations, vol. 198 of Mathematics in Science and Engineering, Academic Press, San Diego, Calif, USA, 1999.

[18] I. S. Gradshteyn and I. M. Ryzhik, Table of Integrals, Series, and Products, Academic Press, New York, NY, USA, 1965.

[19] M. Abramowitz and I. A. Stegun, Handbook of Mathematical Functions With Formulas, Graphs And Mathematical Table, vol. 14, U.S. Department of Commerce, Washington, DC, USA, 1964, Table Errata Math. Comput. vol. 21, p. 747, 1964.

[20] NIST Digital Library of Mathematical Functions, Release 1.0.8 of 2014-04-25, http://dlmf.nist.gov/.

[21] F. W. J. Olver, D. W. Lozier, R. F. Boisvert, and C. W. Clark, Eds., NIST Handbook of Mathematical Functions, Cambridge University Press, Cambridge, UK, 2010.

[22] A. Buică, Periodic Solutions for Nonlinear Systems, Cluj University Press, Cluj-Napoca, Romania, 2006.

[23] T. A. Burton, Stability and Periodic Solutions of Ordinary and Functional Differential Equations, Dover Publications, Mineola, NY, USA, 2005.

[24] E. Reithmeier, Periodic Solutions of Nonlinear Dynamical Systems, vol. 1483 of Lecture Notes in Mathematics, Springer, Berlin, Germany, 1991.

[25] M. Belmekki, J. J. Nieto, and R. Rodrguez-López, "Existence of periodic solution for a nonlinear fractional differential equation," Boundary Value Problems, vol. 2009, Article ID 324561, 18 pages, 2009. 


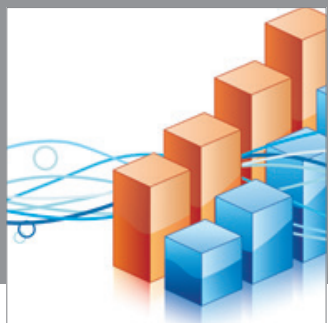

Advances in

Operations Research

mansans

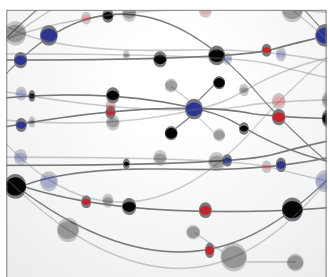

The Scientific World Journal
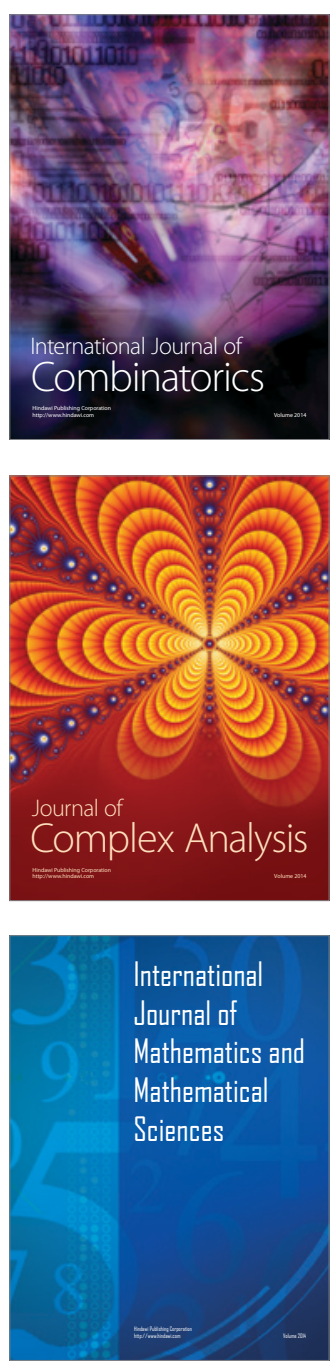
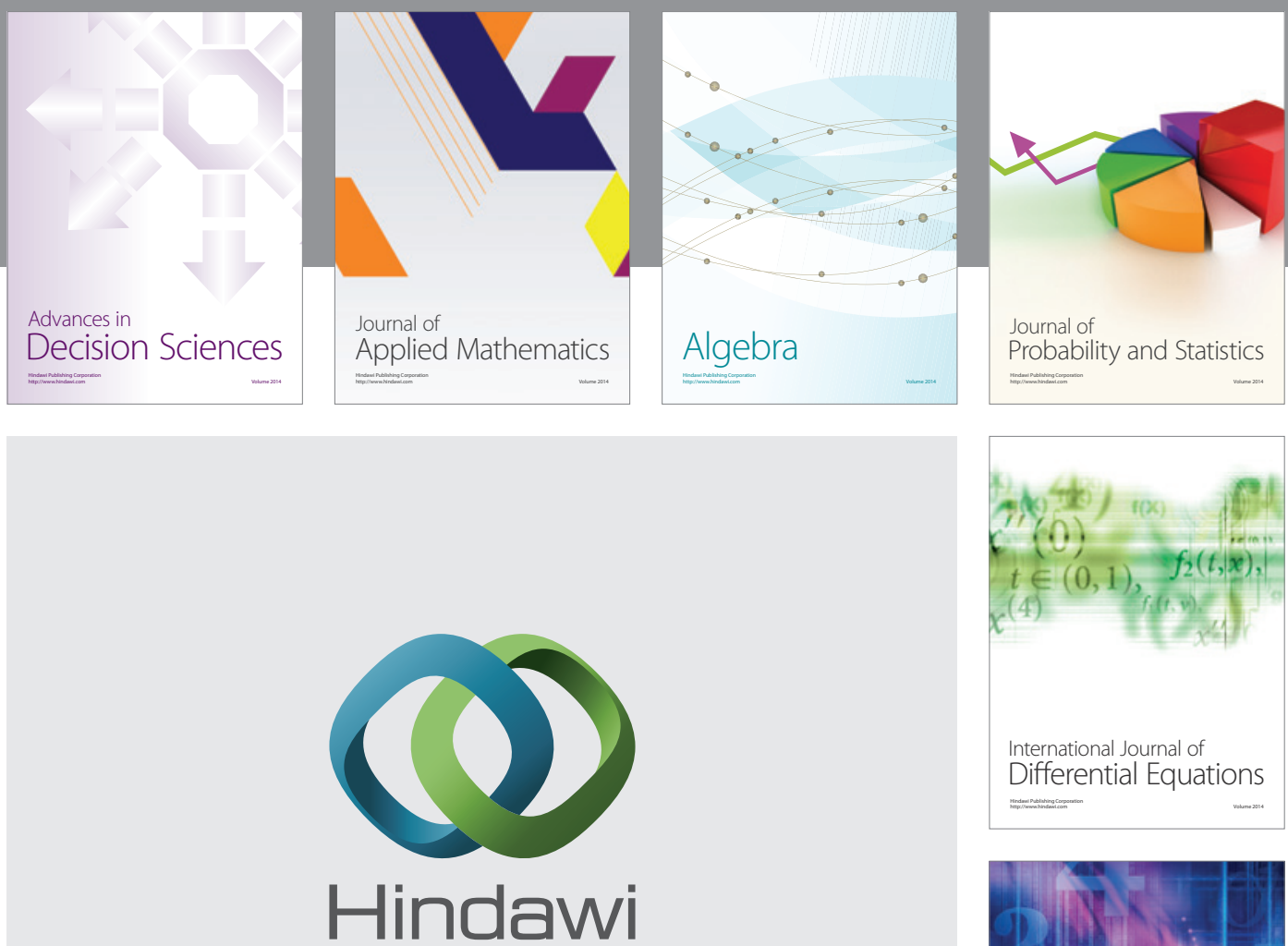

Submit your manuscripts at http://www.hindawi.com
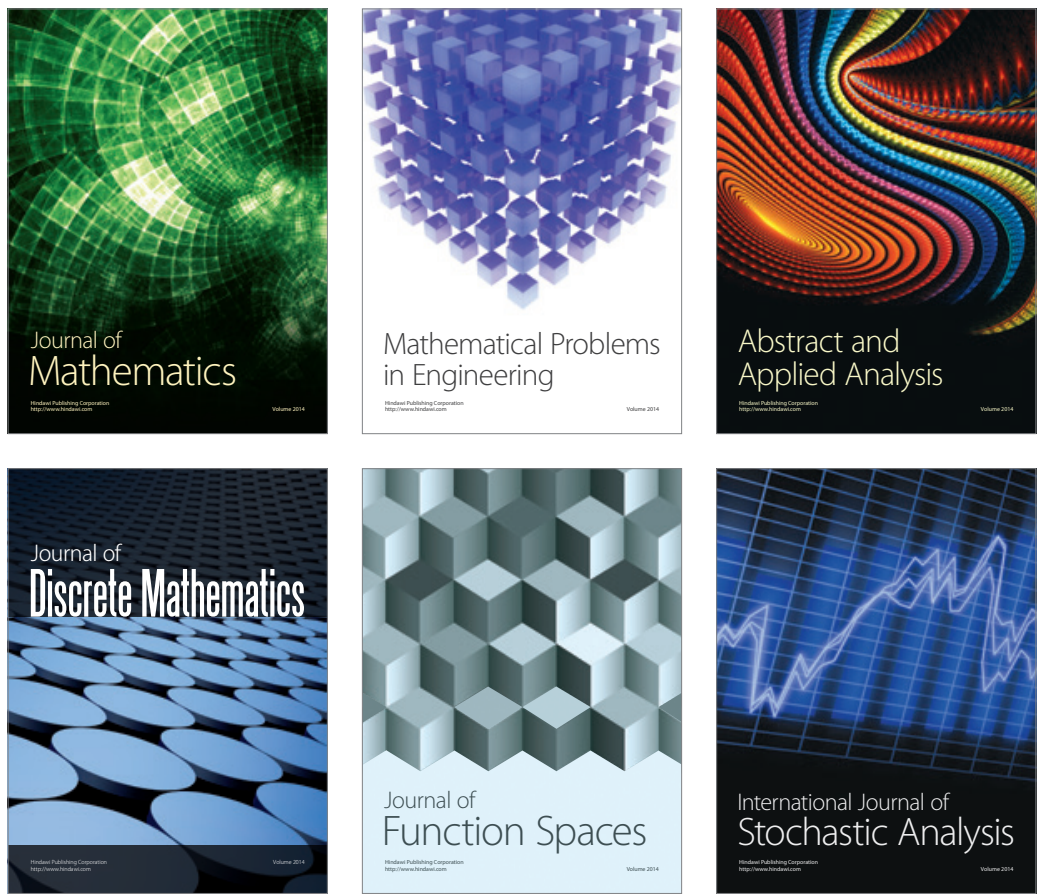

Journal of

Function Spaces

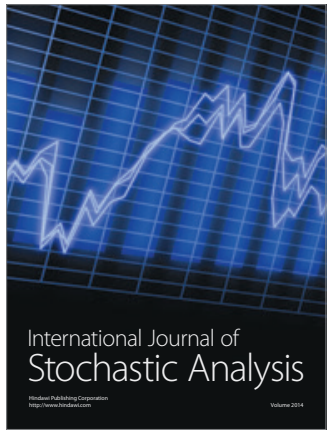

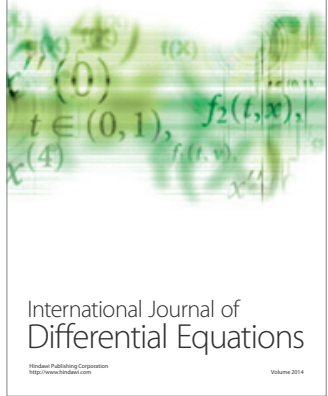
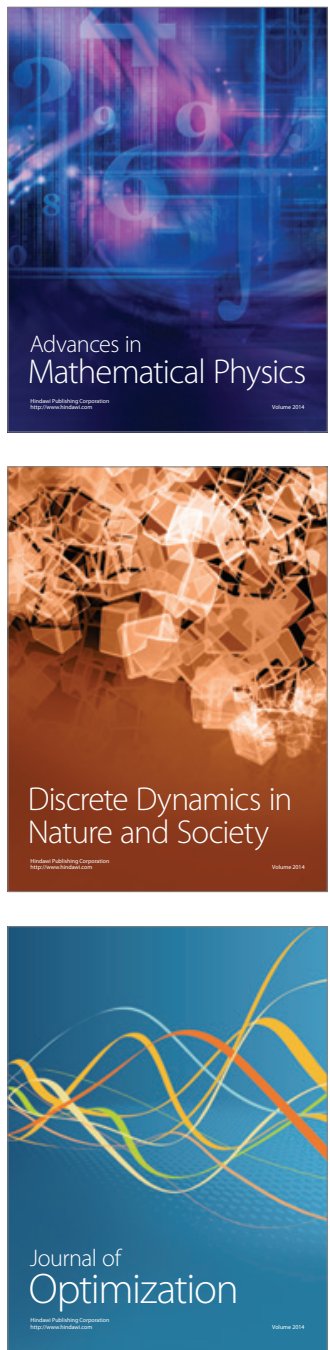